\title{
Restricted mean survival time of older adults with severe aortic stenosis referred for transcatheter aortic valve replacement
}

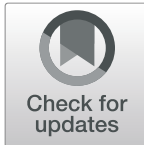

Julia Rodighiero ${ }^{1}$ D, Nicolo Piazza², Giuseppe Martucci², Marco Spaziano², Kevin Lachapelle³, Benoit de Varennes ${ }^{3}$, Marie-Claude Ouimet ${ }^{1}$ and Jonathan Afilalo ${ }^{1,4^{*}}$

\begin{abstract}
Background: Few studies have measured frailty as a potential reason for foregoing transcatheter aortic valve replacement (TAVR) in older adults with severe aortic stenosis (AS). This study sought to determine the impact of frailty and other clinician-cited reasons on restricted mean survival time (RMST).

Methods: An analysis of the McGill Frailty Registry was conducted between 2014 and 2018 at the McGill University Health Center Structural Valve Clinic. Consecutive nonsurgical patients referred for TAVR were included. In those that underwent balloon aortic valvuloplasty or medical management, the primary clinician-cited reason for foregoing TAVR was codified. Vital status was ascertained at 1 year and analysed using RMST and Kaplan-Meier analyses.

Results: The study consisted of 373 patients with a mean age of 82.4 years, of which 233 underwent TAVR and 140 did not. Patients who did not undergo TAVR were more likely to be nonagenarians, with left ventricular dysfunction, chronic kidney disease, dementia, disability, depression, malnutrition, and frailty. The primary cliniciancited reason was: comorbidity in 34\%, frailty in $23 \%$, procedural feasibility and risks in $16 \%$, and mild or unrelated symptoms in $27 \%$. Compared to the TAVR group, 1-year RMST was reduced by 2.0 months in the medical management group $(95 \% \mathrm{Cl} 1.2,2.7)$ and by 1.1 months in the valvuloplasty group $(95 \% \mathrm{Cl}-0.2,2.5)$.

Conclusions: Patients with severe AS referred for TAVR may never undergo the procedure on the basis of comorbidity, frailty, procedural issues, and symptoms. The best treatment decision is one that follows from multidisciplinary assessment encompassing frailty.
\end{abstract}

Keywords: Aortic stenosis, TAVR, Frailty, Survival

\footnotetext{
* Correspondence: jonathan.afilalo@mcgill.ca

${ }^{1}$ Research Institute of the McGill University Health Centre, Montreal, QC,

Canada

${ }^{4}$ Division of Cardiology and Centre for Clinical Epidemiology, Jewish General Hospital, McGill University, 3755 Cote Ste Catherine Road, Montreal, QC H3T 1E2, Canada

Full list of author information is available at the end of the article
}

(c) The Author(s). 2020 Open Access This article is licensed under a Creative Commons Attribution 4.0 International License, which permits use, sharing, adaptation, distribution and reproduction in any medium or format, as long as you give appropriate credit to the original author(s) and the source, provide a link to the Creative Commons licence, and indicate if changes were made. The images or other third party material in this article are included in the article's Creative Commons licence, unless indicated otherwise in a credit line to the material. If material is not included in the article's Creative Commons licence and your intended use is not permitted by statutory regulation or exceeds the permitted use, you will need to obtain permission directly from the copyright holder. To view a copy of this licence, visit http://creativecommons.org/licenses/by/4.0/. The Creative Commons Public Domain Dedication waiver (http://creativecommons.org/publicdomain/zero/1.0/) applies to the data made available in this article, unless otherwise stated in a credit line to the data. 


\section{Background}

Approximately $8 \%$ of individuals 80 years or older are affected by calcific aortic valve stenosis (AS), with the prevalence expected to grow in parallel to the aging population demographics [1]. Given the pathophysiological link between AS, aging, and cardiovascular risk factors, patients with AS often present at advanced ages suffering from multiple cardiac and non-cardiac comorbidities. In the past, one-third of patients were judged to be too old or comorbid to undergo surgical aortic valve replacement [2], and were therefore managed with palliative medical therapy. More recently, transcatheter aortic valve replacement (TAVR) has emerged as a less invasive option that was shown to be safe and superior to medical therapy for very high-risk older patients with severe symptomatic AS. The advent of TAVR has broadened the eligible patient population to include the oldest old who were previously not candidates. Even in nonagenarians, the procedural success rate has exceeded 95\% and the 30-day and 1-year mortality rates have been estimated to be $5.5 \%$ and $23.0 \%$, respectively [3]. However, despite these encouraging metrics, certain patients are evaluated and ultimately judged not to be good candidates for the TAVR procedure. The reasons may be multi-factorial owing to procedural considerations, comorbidities, and frailty. Few studies have systematically addressed the reasons for not proceeding with TAVR, and none have prospectively measured frailty using objective criteria as a key potential reason. Therefore, we sought to compare patients who proceeded with TAVR with those that did not to better understand the reasons cited by clinicians and patients for arriving at their decision and to determine the impact of these reasons on subsequent survival.

\section{Methods}

\section{Study design}

An analysis of the McGill Frailty Registry was conducted between 2014 and 2018 at the McGill University Health Center (Montreal, Quebec) clinic for structural heart valve disease. The Registry prospectively captures consecutive patients referred and evaluated for surgical or transcatheter heart procedures, including TAVR. In addition to the usual clinic assessment, a trained research assistant administers a comprehensive questionnaire and physical performance battery focused on frailty and other geriatric domains, and shares the results of these tests with the treating clinicians to help guide decision making. After the clinic visit and heart team discussion, a research assistant reviews the medical records to determine and codify the final treatment decision and justification, and they contact the patients to ascertain vital status at 1 year.

\section{Patient population}

Inclusion criteria were (1) severe AS, (2) age $\geq 60$ years, (3) signed informed consent to participate in the Registry, (4) clinical evaluation in the structural heart valve clinic for consideration of TAVR, (5) did not proceed with TAVR or cardiac surgery within 1-year of the index clinic visit. Patients who underwent TAVR during the study time frame were retained for comparative analyses. Patients who underwent balloon aortic valvuloplasty (BAV) were considered as part of the no TAVR group, unless they underwent subsequent TAVR within 1 year in which case, they were considered as part of the TAVR group. Exclusion criteria were (1) proceeded with surgical aortic valve replacement, and (2) referred for consideration of mitral, tricuspid, or pulmonic valve procedures.

\section{Clinical assessments}

The clinic visit consisted of a multi-disciplinary assessment by a nurse practitioner, a structural cardiologist, and a research assistant from the FRAILTY team. The research assistant assessed the Essential Frailty Toolset (EFT) [4] for lower-extremity strength, cognitive function, albumin, hemoglobin, as well as assessments for upper-extremity strength, gait speed, balance, social support, depression, and disability for basic and instrumental activities of daily living. The patient underwent an electrocardiogram, echocardiogram, and if the clinician and patient were ready to consider TAVR, they underwent a computed tomography scan, coronary angiogram, and heart team discussion to integrate all of the information and arrive at a treatment decision.

\section{Reason for not proceeding with TAVR}

When the decision not to proceed with TAVR was final, a research assistant ascertained the primary reason as documented by the treating clinician or the patient in cases when the latter refused intervention. These reasons were categorized as follows: (a) patient's cardiac or extra-cardiac comorbidities, (b) patient's frailty, (c) patient's low symptom burden or symptoms not attributable to AS, (d) procedure's technical feasibility or risks. In cases when more than one reason was cited, the predominant reason was chosen based on that which was most strongly or directly stated in the medical records. When uncertain, the research assistant asked the treating clinician to clarify.

\section{Statistical approach}

Baseline characteristics and frailty traits were summarized with proportions, means and standard deviations and compared between TAVR and no TAVR groups using chi-squared tests and t-tests. One-year survival was compared between TAVR, no intervention, and 
BAV groups using (i) Kaplan-Meier analysis, (ii) Cox proportional hazards analysis adjusted for the Society of Thoracic Surgeons Predicted Risk of Mortality (STSPROM) and the EFT score, and (iii) restricted mean survival time (RMST). The RMST indicates the average survival time over a pre-specified time frame, reflecting the differences in areas under Kaplan-Meier curves for patient groups. The RMST is not dependent on the proportional hazards assumption, and it reflects the entire time frame rather than instantaneous time points [5]. In the no TAVR group, 1-year survival was compared between subgroups of pre-defined reasons for not proceeding with TAVR. Statistical analyses were performed with STATA version 16 (College Station, TX).

\section{Results}

\section{Baseline cohort characteristics}

The study consisted of 373 nonsurgical AS patients evaluated in the structural heart valve disease clinic between 2014 and 2018, of which 233 proceeded with TAVR and 140 did not (Fig. 1). Overall, the mean age was 82.4 \pm 6.6 years and the mean STS-PROM was $4.4 \pm 3.0 \%$. Patients who did not proceed with TAVR had similar STS-
PROM scores although they were older, more likely to be nonagenarians, more likely to have left ventricular dysfunction, chronic kidney disease, cognitive impairment, disability for activities of daily living, depression, malnutrition, and frailty according to various scales (Tables 1 and 2). The mean EFT score was $2.3 \pm 1.1$ vs. $1.6 \pm 1.1$ out of 5 in patients who did not proceed vs. proceeded with TAVR, respectively $(P<0.001)$.

\section{Reasons for not proceeding with TAVR}

Among 140 patients who did not proceed with TAVR, the primary cited reason was: comorbidity in $34 \%$, frailty in $23 \%$, procedural feasibility and risks in $16 \%$, and mild or unrelated symptoms in $27 \%$. When frailty was cited by the clinician as the primary reason, there was objective evidence of physical or psycho-social frailty in $94 \%$ of cases as measured by either the EFT, Clinical Frailty Scale, Fried scale, or disability for basic activities of daily living (with the remaining 6\% being subjective impressions). Although comorbidity was often cited by the clinician, only chronic kidney disease was consistently predictive of no TAVR whereas many other comorbidities were more frequent in TAVR patients. A total of 16

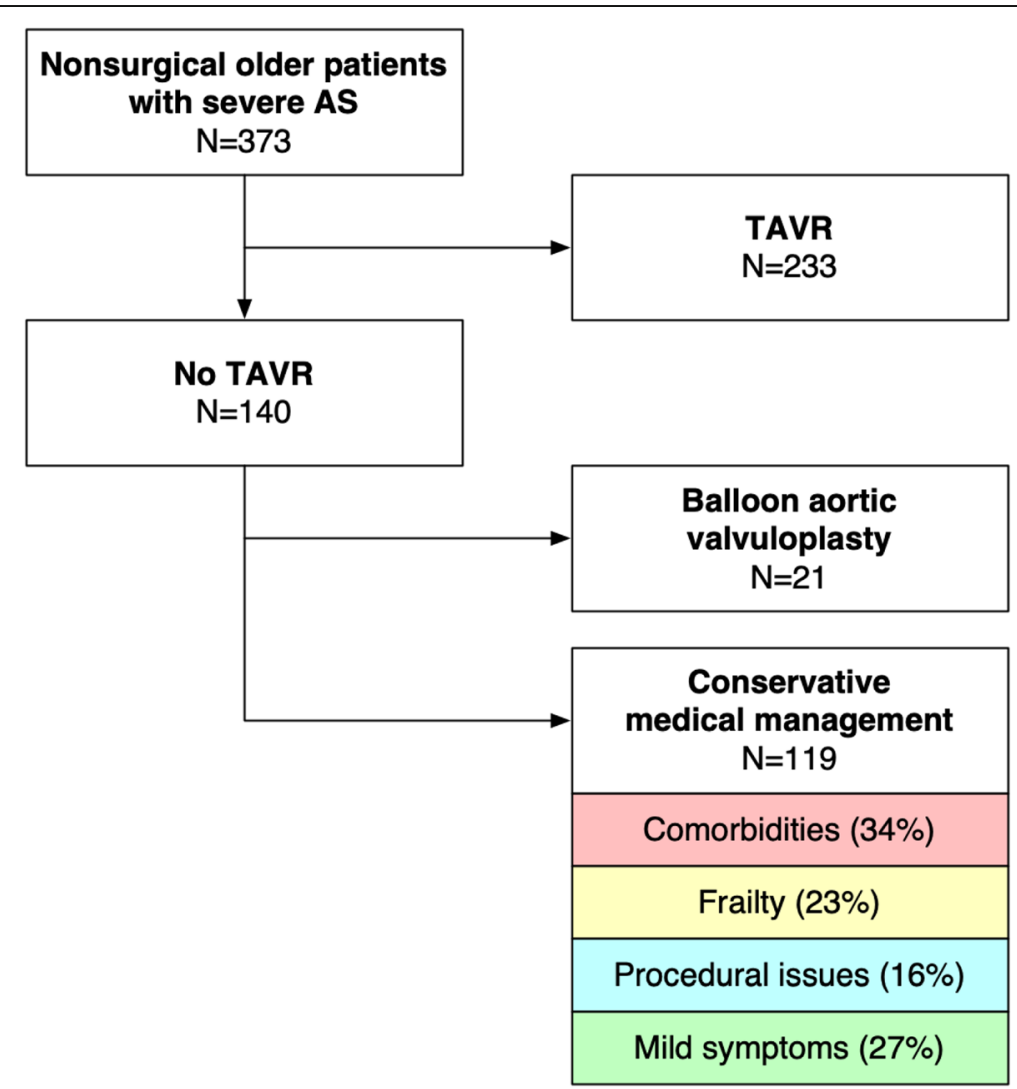

Fig. 1 Flow Diagram. Abbreviations: AS, aortic stenosis; TAVR, transcatheter aortic valve replacement. Legend: A total of 373 older adults with severe AS were included: 233 underwent TAVR and 140 did not undergo TAVR. The primary cited reason for not undergoing TAVR was either comorbidities, frailty, procedural feasibility and risks, or mild or non-AS-related symptoms 
Table 1 Clinical Characteristics

\begin{tabular}{lllll}
\hline & $\begin{array}{l}\text { Total } \\
(N=373)\end{array}$ & $\begin{array}{l}\text { TAVR } \\
(n=233)\end{array}$ & $\begin{array}{l}\text { No TAVR } \\
(n=140)\end{array}$ & $P$-value \\
\hline DEMOGRAPHICS & & & & \\
Age, years & $82.4 \pm 6.6$ & $81.4 \pm 6.3$ & $84 \pm 6.6$ & $<0.001$ \\
Female sex & $184(49 \%)$ & $108(46 \%)$ & $76(54 \%)$ & 0.14 \\
Body Mass Index, kg/m2 & $27.1 \pm 6$ & $27.4 \pm 6.1$ & $26.3 \pm 5.7$ & 0.12 \\
COMORBIDITIES & & & & \\
Diabetes mellitus & $80(21 \%)$ & $66(28 \%)$ & $14(10 \%)$ & $<0.001$ \\
Coronary artery disease & $196(53 \%)$ & $137(59 \%)$ & $59(42 \%)$ & 0.002 \\
Prior myocardial infarction & $38(10 \%)$ & $26(11 \%)$ & $12(9 \%)$ & 0.42 \\
Prior stroke & $19(5 \%)$ & $17(7 \%)$ & $2(1 \%)$ & 0.01 \\
Peripheral arterial disease & $24(6 \%)$ & $19(8 \%)$ & $5(4 \%)$ & 0.08 \\
Chronic kidney disease & $187(50 \%)$ & $104(45 \%)$ & $83(59 \%)$ & 0.006 \\
Dialysis-dependent & $7(2 \%)$ & $5(2 \%)$ & $2(2 \%)$ & 0.85 \\
Cirrhosis & $7(2 \%)$ & $5(2 \%)$ & $2(1 \%)$ & 0.62 \\
NYHA class & $2.5 \pm 0.6$ & $2.4 \pm 0.6$ & $2.5 \pm 0.7$ & 0.15 \\
ECHOCARDIOGRAM & & & & \\
LVEF $\leq 35 \%$ & $34(9 \%)$ & $15(7 \%)$ & $19(14 \%)$ & 0.02 \\
PASP $\geq 60$ mmHg & $50(13 \%)$ & $27(12 \%)$ & $23(16 \%)$ & 0.18 \\
Mean aortic gradient, mmHg & $49.5 \pm 18.3$ & $52 \pm 17.7$ & $45.4 \pm 18.6$ & $<0.001$ \\
\hline
\end{tabular}

Abbreviations: $L V E F$ left ventricular ejection fraction, NYHA New York Heart Association, PASP pulmonary artery systolic pressure, TAVR transcatheter aortic valve replacement patients died during the TAVR work-up period or while waiting for TAVR; most often patients who had been referred for additional testing or optimization of severe comorbidities before possible TAVR.

\section{Comparison of TAVR, BAV, and no intervention groups}

The Kaplan-Meier survival curves stratified by TAVR, BAV, and no intervention are shown in Fig. 2. RMST during the first year of follow-up was 11.6 months in the TAVR group (95\% CI 11.3, 11.8), 10.4 months in the BAV group (95\% CI 9.1, 11.7 months), and 9.6 months in the no intervention group $(95 \% \mathrm{CI} 8.8,10.3)$. Compared to the TAVR group, RMST was reduced by 1.1 months in the BAV group $(95 \% \mathrm{CI}-0.2,2.5)$ and by 2.0 months in the no intervention group (95\% CI 1.2, 2.7). After adjusting for STS-PROM and EFT scores, there remained a trend towards reduced survival in the BAV group (HR 1.82; 95\% CI 0.72, 4.60) and a significant reduction in the no intervention group (HR 4.59; 95\% CI 2.61, 8.04). Inspection of the Kaplan-Meier curves showed that survival in the BAV group was relatively preserved up until 8 months post-procedure, after which time it declined to the level of the no intervention group.

\section{Comparison of reasons for not proceeding with TAVR}

The Kaplan-Meier survival curves stratified by comorbidity, frailty, procedural feasibility and risk, and mild or unrelated symptoms are shown in Fig. 3. RMST during the first year of follow-up was 8.3 months in the comorbidity category (95\% CI 7.0, 9.6), 9.5 months in the frailty

Table 2 Geriatric Characteristics

\begin{tabular}{|c|c|c|c|c|}
\hline & Total $(N=373)$ & $\operatorname{TAVR}(n=233)$ & No TAVR $(n=140)$ & $P$-value \\
\hline \multicolumn{5}{|l|}{$\overline{\text { FRAILTY }}$} \\
\hline Essential Frailty Toolset & $92(26 \%)$ & $43(19 \%)$ & 49 (40\%) & $<0.001$ \\
\hline Fried Frailty scale & $99(27 \%)$ & 45 (19\%) & $54(39 \%)$ & $<0.001$ \\
\hline Rockwood Clinical Frailty Scale & $98(26 \%)$ & $37(16 \%)$ & $61(44 \%)$ & $<0.001$ \\
\hline \multicolumn{5}{|l|}{ PHYSICAL DOMAINS } \\
\hline Slow chair rise time & $284(76 \%)$ & $158(68 \%)$ & $126(91 \%)$ & $<0.001$ \\
\hline Slow gait speed & $215(58 \%)$ & $112(48 \%)$ & $103(74 \%)$ & $<0.001$ \\
\hline Weak grip strength & $194(52 \%)$ & 103 (44\%) & $91(65 \%)$ & $<0.001$ \\
\hline Fall(s) & $20(5 \%)$ & $13(6 \%)$ & $7(5 \%)$ & 0.81 \\
\hline \multicolumn{5}{|l|}{ NON-PHYSICAL DOMAINS } \\
\hline ADL limitation & $167(45 \%)$ & 79 (34\%) & $88(63 \%)$ & $<0.001$ \\
\hline Living in assisted facility & $59(16 \%)$ & $33(14 \%)$ & $26(19 \%)$ & 0.26 \\
\hline Cognitive impairment & $47(13 \%)$ & $18(8 \%)$ & 29 (21\%) & $<0.001$ \\
\hline Depression & $88(24 \%)$ & 45 (19\%) & $43(31 \%)$ & 0.01 \\
\hline Malnourishment & $32(9 \%)$ & $10(4 \%)$ & $22(16 \%)$ & $<0.001$ \\
\hline
\end{tabular}

Abbreviations: TAVR transcatheter aortic valve replacement

Legend: Cut-offs for various domains were $\geq 3 / 5$ for Essential Frailty Toolset, $\geq 3 / 5$ for Fried Frailty Scale, $\geq 5 / 9$ for Rockwood Clinical Frailty Scale, $\geq 15 \mathrm{~s}$ for slow chair rise time, $\geq 0.83 \mathrm{~m} / \mathrm{second}$ for slow gait speed, $<30 \mathrm{~kg}$ in men and $<20 \mathrm{~kg}$ in women for weak grip strength, $<24 / 30$ for Mini-Mental State Examination (cognitive impairment), $\geq 2 / 5$ for Geriatric Depression Scale, $<8 / 14$ for Mini-Nutritional Assessment 


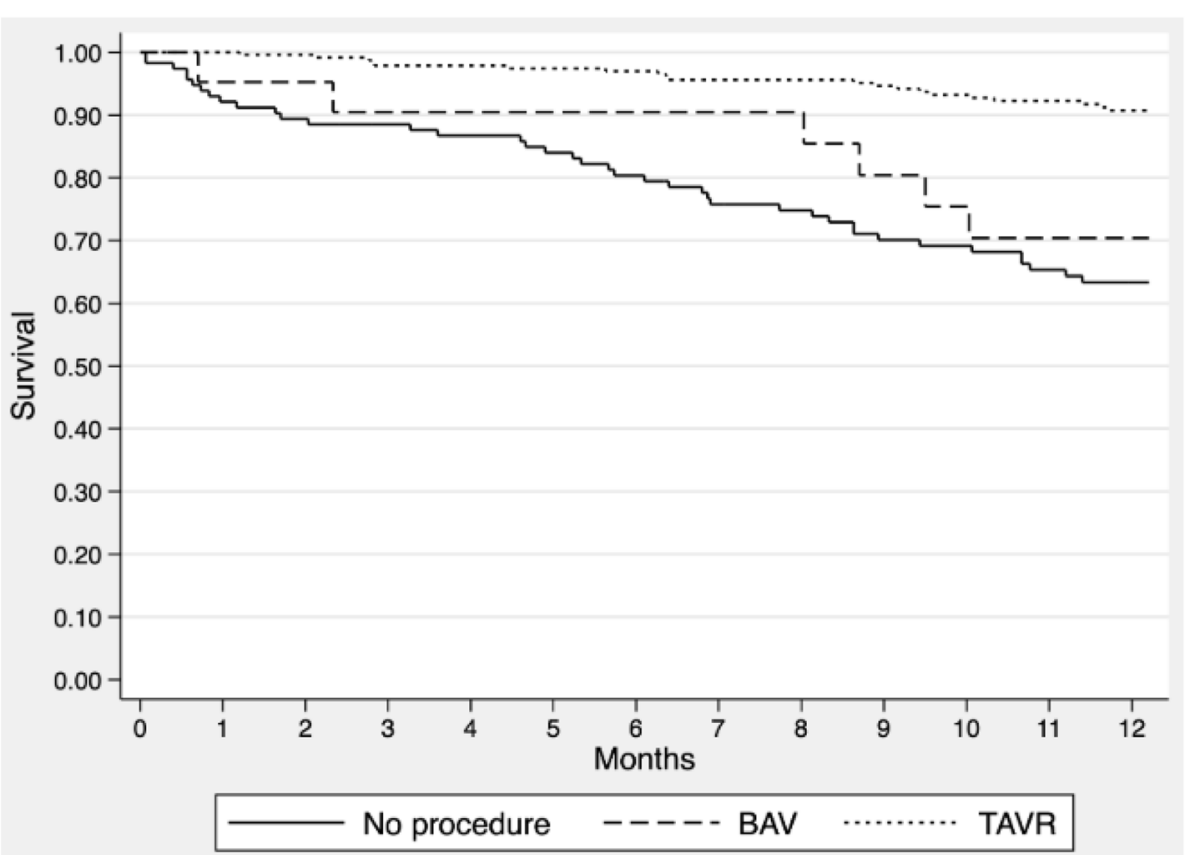

Fig. 2 Survival by Treatment Group. Legend: Kaplan-Meier survival curves for patients referred to our clinic stratified by TAVR, BAV, and conservative medical management. Abbreviations as in Fig. 1

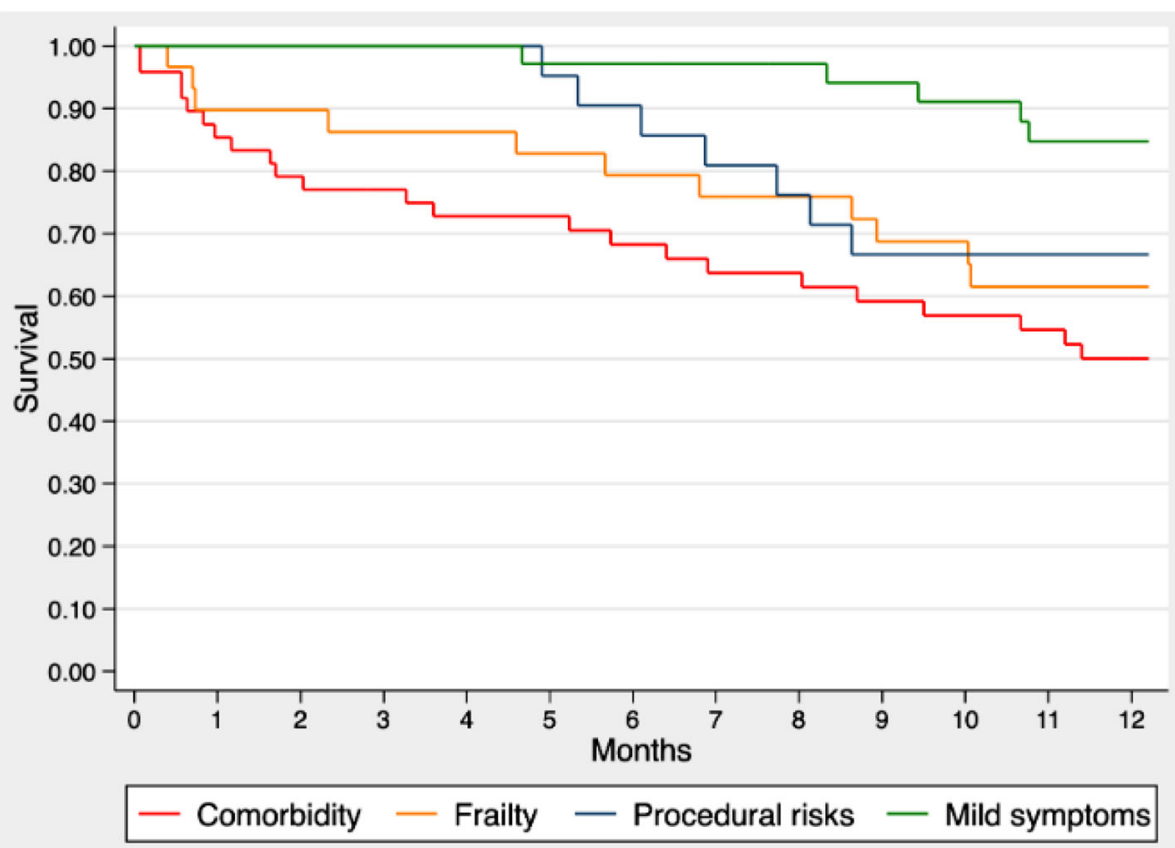

Fig. 3 Survival by Clinician-Cited Reason for Foregoing TAVR. Legend: Kaplan-Meier survival curves for patients not undergoing TAVR, stratified by the clinician-cited primary reason for which TAVR was not performed. Abbreviations as in Fig. 1 
category (95\% CI 7.9, 11.1 months), 10.3 months in the procedural feasibility and risk category (95\% CI 9.0, 11.6 months), and 11.5 months in the mild or unrelated symptoms category (95\% CI 10.8, 12.3). Compared to the mild or unrelated symptoms category, RMST was significantly reduced by 3.2 months in the comorbidity category $(95 \% \mathrm{CI} 1.7,4.7)$ and by 2.0 months in the frailty category $(95 \%$ CI $0.3,3.8)$. Adjustment for STSPROM and EFT scores was not performed since risk and frailty were embedded in the clinicians' cited reasons.

\section{Discussion}

This study from the McGill Frailty Registry examined the factors influencing clinical decision making and ensuing survival among older AS patients who received TAVR, BAV, or conservative medical management. The lessons learned can be summarized as follows. First, TAVR was associated with a gain in projected survival of 2 months within the first year as compared to conservative medical management. Second, BAV was associated with a projected survival similar to TAVR for approximately 8 months, after which time survival (in the absence of definitive TAVR) declined precipitously. Third, the primary reason for not proceeding with TAVR was cited as either comorbidity or frailty, and these patients had the highest mortality rates. Lastly, when the primary reason for not proceeding was mild or unrelated symptoms, these patients had relatively low mortality rates during the first year of follow-up.

Previous studies have described reasons for not proceeding with TAVR [6-8], however, these studies focused on comorbidities and symptoms and did not benefit from systematically-performed objective evaluations of frailty. This study further adds to the existing body of knowledge by connecting the various reasons for not proceeding with TAVR with projected survival. While comorbidity and frailty were associated with the lowest survival, deferral due to mild symptoms was associated with higher relative survival. Nevertheless, the 1year mortality rate for mildly symptomatic patients was non-negligible, estimated at $15 \%$, supporting the trend to perform aortic valve replacement sooner rather than later when AS is very severe - potentially even in asymptomatic patients [9].

An especially poor prognosis was observed when the cited reason for not proceeding with TAVR was comorbidities. Specific comorbidities found to be prevalent and influential for decision making were reduced LVEF, severe chronic kidney disease, and dementia; consistent with prior studies [10]. In a report from the STS/ACC TVT Registry, dialysis-dependent kidney disease was associated with a twofold increase in 1-year mortality rate up to $37 \%$ [11]. Other studies have reaffirmed the substantial increase in long-term and short-term risks $[12,13]$, including the procedural risk of contrastinduced acute kidney injury. In a meta-analysis, cirrhosis was also associated with a twofold increase in 1-year mortality [14]. In an analysis from the CoreValve Trial, oxygen-dependent lung disease was the comorbidity most strongly associated with 1-year mortality [15]. Overall, these data point to comorbidity profiles that may be equated with prohibitive risk or limited benefits to be expected from TAVR.

While frailty was commonly cited as a reason for choosing conservative medical management in our study and a previous study [16], frailty should not be equated with futility. It is important to note that one-half of TAVR patients manifest objective evidence of frailty at baseline, and that most still benefit from the procedure [17]. Those that typically do not benefit have severe frailty as defined by an EFT score of 5/5, advanced dementia, bedbound or non-mobile status, cachexia or severe sarcopenia, or disability for most basic activities of daily living. These risk factors, coupled with the aforementioned end-stage kidney, lung, and liver disease, have been grouped in the A-B-C-D-E mnemonic, which was put forth in the 2019 Canadian Cardiovascular Society TAVR Consensus Statement to guide decision making in potentially futile cases [18].

One of the strengths of this study is the use of the RMST to compare survival times in different subsets of patients who underwent or did not undergo TAVR. The RMST analysis yields results that are not only more intuitive to interpret, but are also more accurate for scenarios in which the hazard of an event changes over time $[5,19]$. Cox proportional hazards analysis assumes that the hazard of an event is constant over the entire follow-up period, while logistic regression analysis assumes that the follow-up time period is constant and fixed for all patients; neither of which would have been ideal in this patient population. In the PARTNER B Trial, RMST was similarly used to demonstrate a gain in projected survival of 12.6 months within 5 years with TAVR as compared to conservative medical management [19].

BAV is used as a temporizing procedure to bridge the gap between TAVR and conservative medical management in challenging cases when patients suffer from severe comorbidities, frailty, or questionable symptoms. Epidemiologic and echocardiographic studies have shown the limited durability of the BAV procedure, with restenosis expected to occur at 6 months as evidenced by a mean aortic valve area progressing from 0.78 to $0.65 \mathrm{~cm}^{2}$ [20]. Once restenosis occurs, the long-term survival approaches the natural history of severe AS patients [20-22]; a finding which is supported by our data. Thus, our results and those of others suggest that 
patients should be reassessed at $6-8$ months post-BAV to reconsider a more definitive procedure if clinically appropriate and in-line with the patient's preferences.

\section{Limitations}

Firstly, this was a single-centre study at an academic university hospital, therefore the reasons for not performing TAVR may not be generalizable to other centres with different practice patterns. Secondly, cause of death was not recorded in this study, although it would have been of interest to examine the distribution of cardiac and non-cardiac causes of deaths in our patient subgroups. Thirdly, time of follow-up was affected by immortaltime in the TAVR and BAV subgroups, since these patients necessarily survived to the time of their procedure while (a small number of) the remaining patients died early before any procedure could be performed. The likelihood of bias was minimized [23] by using the same time zero for all patients, which was defined as the date of the initial structural heart valve disease clinic visit. Fourthly, the RMST does not represent the true benefits of TAVR relative to BAV over a modest 1-year time period. The RMST is known to be skewed in scenarios with few early events [5]. Finally, this study was not designed to define indications for proceeding with TAVR or other treatment strategies; rather, to clearly understand the reasons and outcomes of patients not proceeding with this procedure.

\section{Conclusions}

Patients with severe AS referred for TAVR may never undergo the procedure based on concerns surrounding comorbidity, frailty, procedural feasibility and risks, and mild or unrelated symptoms. These concerns - elicited by clinicians or patients - should be thoroughly investigated and discussed using a shared-decision making approach [24]. A comprehensive treatment decision is one that follows from multi-disciplinary assessment, including objective assessment of frailty and disability, which informs the expected risks and benefits in an individualized fashion. Structured decision aid tools have been developed and validated to facilitate this process [25].

\section{Abbreviations}

TAVR: Transcatheter aortic valve replacement; AS: Aortic stenosis; RMST: Restricted mean survival time; EFT: Essential frailty toolset; STSPROM: Society of Thoracic Surgeons Predicted Risk of mortality; BAV: Balloon aortic valvuloplasty; LVEF: Left ventricular ejection fraction; NYHA: New York heart association; PASP: Pulmonary artery systolic pressure

\section{Acknowledgements}

We would like to thank Dr. Dae H. Kim (Harvard University, Boston, MA) for his guidance on RMST analyses.

\section{Authors' contributions}

JR collected and organized patient data regarding the reasons for TAVR deferral and was a major contributor in writing the manuscript. NP, GM and MS provided data for the analysis. $\mathrm{KL}$ and BDV provided data for the analysis.
MCO determined which patients within the study population did not undergo TAVR. JA statistically interpreted and analysed all collected data and was a major contributor in writing the manuscript. All authors read and approved the final manuscript.

\section{Funding}

The Canadian Institutes of Health Research (CIHR) funded the FRAILTY-AVR study from which some of the patients in this study were recruited.

\section{Availability of data and materials}

The datasets analysed during the current study may be made available from the $\mathrm{PI}$ on reasonable request.

Ethics approval and consent to participate

This study involved human participants and was granted approval was by the McGill University Health Centre Research Ethics Board (reference number: FWA00000840- FWA00000845.

IRB00010120, study number: 2013-2097).

Consent for publication

Not applicable.

\section{Competing interests}

Rodighiero J, none; Piazza, none; Martucci, none; Spaziano, none; Lachapelle, none; de Varennes, none; Ouimet, none; Afilalo, none.

The authors declare that they have no competing interests.

\section{Author details}

${ }^{1}$ Research Institute of the McGill University Health Centre, Montreal, QC, Canada. ${ }^{2}$ Division of Cardiology, McGill University Health Centre, Montreal, QC, Canada. ${ }^{3}$ Division of Cardiac Surgery, McGill University Health Centre, Montreal, QC, Canada. ${ }^{4}$ Division of Cardiology and Centre for Clinical Epidemiology, Jewish General Hospital, McGill University, 3755 Cote Ste Catherine Road, Montreal, QC H3T 1E2, Canada.

Received: 3 April 2020 Accepted: 3 June 2020

Published online: 18 June 2020

\section{References}

1. Transcatheter Aortic Valve Replacement | CADTH.ca. https://cadth.ca/ transcatheter-aortic-valve-replacement-3. Accessed January 15, 2020.

2. Leon $M B$, et al. Transcatheter aortic-valve implantation for aortic stenosis in patients who cannot undergo surgery. N Engl J Med. 2010;363:1597-607.

3. Galatas C, Afilalo J. Transcatheter aortic valve replacement over age 90: risks vs benefits. Clin Cardiol. 2019;43:1-7.

4. Afilalo J, et al. Frailty assessment in older adults undergoing Transcatheter or surgical aortic valve replacement: the frailty-AVR study. J Am Coll Cardiol. 2017:70:689-700.

5. Gregson J, et al. Nonproportional hazards for time-to-event outcomes in clinical trials: JACC review topic of the week. J Am Coll Cardiol. 2019;74: 2102-12.

6. Kapadia SR, et al. Characterization and outcome of patients with severe symptomatic aortic stenosis referred for percutaneous aortic valve replacement. J Thorac Cardiovasc Surg. 2009;137:1430-5.

7. Bach DS, et al. Evaluation of patients with severe symptomatic aortic stenosis who do not undergo aortic valve replacement. Circulation. 2009;2: 533-9.

8. Badran A, Vohra H, Livesey S. Unoperated severe aortic stenosis: decision making in an adult UK-based population. Ann R Coll Surg Engl. 2012;94: 416-21.

9. Everett RJ, Clavel M-A, Pibarot P, Dweck MR. Timing of intervention in aortic stenosis: a review of current and future strategies. Heart. 2018;104:2067-76.

10. Kang D, et al. Mortality predictors in patients referred for but not undergoing Transcatheter aortic valve replacement. Am J Cardiol. 2015;116: 919-24.

11. Szerlip M, et al. Transcatheter aortic valve replacement in patients with endstage renal disease. J Am Coll Cardiol. 2019;73:2806-15.

12. Yamamoto $M$, et al. Prognostic value of chronic kidney disease after Transcatheter aortic valve implantation. J Am Coll Cardiol. 2013;62:869-77. 
13. Gupta T, et al. Association of Chronic Kidney Disease with in-hospital outcomes of Transcatheter aortic valve replacement. J Am Coll Cardiol Intv. 2017;10:2050-60.

14. Takagi $\mathrm{H}$, et al. Meta-analysis of impact of liver disease on mortality after transcatheter aortic valve implantation. J Cardiovasc Med. 2019;20:237-44.

15. Hermiller JB, et al. Predicting early and late mortality after Transcatheter aortic valve replacement. J Am Coll Cardiol. 2016;68:343-52.

16. Shimura T, et al. Patients refusing Transcatheter aortic valve replacement even once have poorer clinical outcomes. J Am Heart Assoc. 2018;7: e009195.

17. Piankova P, Afilalo J. Prevalence and prognostic implications of frailty in Transcatheter aortic valve replacement. Cardiol Clin. 2020;38:75-87.

18. Asgar AW, et al. 2019 Canadian cardiovascular society position statement for Transcatheter aortic valve implantation. Can J Cardiol. 2019;35:1437-48.

19. Kim $\mathrm{DH}, \mathrm{Uno} \mathrm{H}$, Wei L-J. Restricted mean survival time as a measure to interpret clinical trial results. JAMA Cardiol. 2017;2:1179-80.

20. Otto CM, et al. Three-year outcome after balloon aortic valvuloplasty. Insights into prognosis of valvular aortic stenosis. Circulation. 1994;89:642-50.

21. Lieberman EB, et al. Balloon aortic valvuloplasty in adults: failure of procedure to improve long-term survival. J Am Coll Cardiol. 1995;26:1522-8.

22. Kapadia $\mathrm{S}$, et al. Outcomes of inoperable symptomatic aortic stenosis patients not undergoing aortic valve replacement: insight into the impact of balloon aortic Valvuloplasty from the PARTNER trial (placement of AoRtic TraNscathetER valve trial). J Am Coll Cardiol Intv. 2015;8:324-33.

23. Platt RW, Hutcheon JA, Suissa S. Immortal time bias in epidemiology. Curr Epidemiol Rep. 2019;6:23-7.

24. Otto CM, et al. 2017 ACC expert consensus decision pathway for Transcatheter aortic valve replacement in the Management of Adults with aortic stenosis: a report of the American College of Cardiology Task Force on clinical expert consensus documents. J Am Coll Cardiol. 2017;69:1313-46.

25. Aortic Stenosis: TAVR/SAVR. https://www.cardiosmart.org/SDM/DecisionAids/Find-Decision-Aids/Aortic-Stenosis. Accessed 17 Jan 2020.

\section{Publisher's Note}

Springer Nature remains neutral with regard to jurisdictional claims in published maps and institutional affiliations.

Ready to submit your research? Choose BMC and benefit from:

- fast, convenient online submission

- thorough peer review by experienced researchers in your field

- rapid publication on acceptance

- support for research data, including large and complex data types

- gold Open Access which fosters wider collaboration and increased citations

- maximum visibility for your research: over $100 \mathrm{M}$ website views per year

At $\mathrm{BMC}$, research is always in progress.

Learn more biomedcentral.com/submissions 